\title{
Kesirli Nötral Diferansiyel Denklemlerin Çözümlerinin Kalitatif Davranışları Üzerine
}

\author{
On The Qualitative Behaviors of Solutions of Fractional Neutral Differential Equations
}

\section{Hakan ADIGÜZEL ${ }^{1}$}

${ }^{i}$ İstanbul Gelişim Üniversitesi, Mekatronik Mühendisliği Bölümü, Avcılar, Istanbul.

\section{$\ddot{O} \mathbf{z}$}

$\mathrm{Bu}$ çalışmada, kesirli nötral diferensiyel denklemlerin bir sınıfı dikkate alınmıştır. Yeni karşılaştırma teoremlerine dayanarak, salınımlılık sonuçları elde edilmiştir. Elde edilen sonuçlar literatürdeki çalışmaları tamamlamış ve genelleştirmiştir.

Anahtar Kelimeler: Salınım, Kesirli Türev, Kesirli Diferensiyel Denklemler, Nötral

\begin{abstract}
In this study, we consider a class of fractional neutral differential equations. Based on new comparison theorems, we obtain some oscillation results. The obtained results complement and improve a number of results in the literature.
\end{abstract}

Keywords: Oscillation, Fractional Derivative, Fractional Differential Equations, Neutral

\section{GÍRIŞ}

Kesirli diferensiyel denklemler çoğu mühendislik probleminin modellenmesinde önemli bir araç olarak karşımıza çıkmaktadır. Özellikle biyomühendislik, elektrokimya, kontrol, elektromanyetik alan teorisi ve daha birçok fiziksel problemlerdeki matematiksel modellerin kurulması gibi geniş bir kullanım alanı vardır [1-2]. Bu anlamda kesirli diferensiyel denklemler hakkında daha fazla bilgi sahibi olunulması istenmesi doğaldır. Bu amaçla son yıllarda bu denklemlerle ilgili çok sayıda çalışma göze çarpmaktadır [3-11]. Bu çalışmalar incelendiğinde özellikle nötral kesirli diferensiyel denklemlerin salınımlılığı konusunda fazla çalışma olmadığı göze çarpmaktadır [12-13].

Wang ve arkadaşları [12],

$$
\left.\begin{array}{l}
\left.D_{t}^{\alpha}\left(a(t)\left[D_{t}^{\alpha}(x(t)+p(t) x(\tau(t)))\right]\right)\right) \\
+q(t) x(\sigma(t))=0
\end{array}\right\}
$$

kesirli diferensiyel denkleminin salınımlılık özelliklerini incelemişlerdir. Ve bu denklem için bazı salınımlılık kriterleri elde etmişlerdir. Ganesan ve arkadaşları [13] ise, Wang ve arkadaşlarının [12] incelediği denklemden daha genel bir denklemi göz önüne alarak verilen salınımlılık kriterlerini daha da genelleştirmişlerdir. 
$\mathrm{Bu}$ çalışmada $z(t)=x(t)+p(t) x(\tau(t))$, $t \geq t_{0}>0$ ve $0<\alpha<1$ olmak üzere,

$$
\left.\begin{array}{rl}
D_{t}^{\alpha}\left(a(t)\left[D_{t}^{\alpha} z(t)\right]^{\gamma}\right) & \\
& +q(t) x^{\beta}(\sigma(t))=0
\end{array}\right\}
$$

denklemi dikkate alınacaktır. Burada $\gamma$ ve $\beta$ iki tek doğal sayının oranını, $D_{t}^{\alpha}$ modifiye Riemann-Lioville kesirli türevini göstermektedir [14]. Ayrıca $q(t) \in C\left(\left[t_{0}, \infty\right)\right)$

$$
D_{t}^{\alpha} a(t) \in C\left(\left[t_{0}, \infty\right)\right),
$$

$D_{t}^{2 \alpha} p(t) \in C\left(\left[t_{0}, \infty\right)\right)$ pozitif fonksiyonlar ve

(1) denklemi

$\left(H_{1}\right) \quad p_{0} \quad$ sabit bir sayı olmak üzere, $0 \leq p(t) \leq p_{0}<\infty$,

$\left(H_{2}\right) \quad \tau^{\prime}(t) \geq \tau_{0}>0, \quad \tau \circ \sigma=\sigma \circ \tau$, $\eta(t) \leq \sigma(t) \quad$ ve $\quad \lim _{t \rightarrow+\infty} \eta(t)=+\infty$ olacak şekilde bir $\eta(t)$ fonksiyonu vardır,

$$
\begin{aligned}
& \left(H_{3}\right) \int_{t_{0}}^{\infty} a^{-1 / \lambda}(s) d s=\infty \\
& \left(H_{4}\right) \frac{t}{\tau(t)} \geq l>0
\end{aligned}
$$

şartlarını sağlar. Modifiye Riemann-Liouville kesirli türevi [14] ve bazı önemli özellikleri aşağıda verilmiştir.

$$
\begin{aligned}
& D_{t}^{\alpha} f(t)=\left\{\begin{array}{c}
\frac{1}{\Gamma(1-\alpha)} \frac{d}{d t} \int_{0}^{t} \frac{f(\xi)-f(0)}{(t-\xi)^{\alpha}} d \xi, \quad 0<\alpha<1 \\
\left(f^{(n)}(t)\right)^{(\alpha-n)}, 1 \leq n \leq \alpha \leq n+1
\end{array}\right. \\
& D_{t}^{\alpha}(f(t) g(t))=g(t) D_{t}^{\alpha} f(t)+f(t) D_{t}^{\alpha} g(t) \\
& \left.\begin{array}{rl}
D_{t}^{\alpha} f[g(t)] & =f_{g}^{\prime}[g(t)] D_{t}^{\alpha} g(t) \\
& =D_{t}^{\alpha} f[g(t)]\left(g^{\prime}(t)\right)^{\alpha}
\end{array}\right\}
\end{aligned}
$$

$$
D_{t}^{\alpha} t^{\beta}=\frac{\Gamma(\beta+1)}{\Gamma(\beta+1-\alpha)} t^{\beta-\alpha}
$$

Genel olarak, (1) denkleminin herhangi çözümü eninde sonunda sonunda pozitif ya da negatif bir çözüm değilse bu çözüme salınımlıdır, denir. Eğer (1) denkleminin tüm çözümleri salınımlı ise (1) denklemine salınımlı denklem denir.

$\mathrm{Bu}$ çalışmada,

$\xi=y(t)=\frac{t^{\alpha}}{\Gamma(1+\alpha)} \quad$ ve $\quad i=0,1 \quad$ için $\xi_{i}=y\left(t_{i}\right)=\frac{t_{i}^{\alpha}}{\Gamma(1+\alpha)} \quad$ olmak üzere $\xi$ değişken dönüşümü kullanılacaktır. $\mathrm{Ve}$ $x(t)=\tilde{x}(\xi), \quad a(t)=\tilde{a}(\xi), p(t)=\tilde{p}(\xi)$, $q(t)=\tilde{q}(\xi)$ olarak göz önüne alınacaktır. Dikkat edilirse $D_{t}^{\alpha} \xi=1$ olacağından Modifiye Riemann-Liouville kesirli türevinin özelliğinden $D_{t}^{\alpha} x(t)=D_{t}^{\alpha} \tilde{x}(\xi)=\tilde{x}^{\prime}(\xi)$ olur ve

$$
\begin{aligned}
D_{t}^{\alpha} x(\tau(t)) & =D_{t}^{\alpha} \tilde{x}(\tilde{\tau}(\xi)) \\
& =(\tilde{x}(\tilde{\tau}(\xi)))^{\prime} D_{t}^{\alpha} \xi(t)=(\tilde{x}(\tilde{\tau}(\xi)))^{\prime}
\end{aligned}
$$

ayrica

yazılır. Benzer şekilde diğer fonksiyonlarda da bu özellik kullanılacaktır.

Kolaylı olması bakımından

$Q(\xi)=\min \{\tilde{q}(\xi), \tilde{q}(\tilde{\tau}(\xi))\} \quad$ ve

$B(t)=\int_{t_{1}}^{t} a^{-1 / \gamma}(s) d s$ olmak üzere

$Q_{v}(\xi)=Q(\xi)[B(\tilde{\eta}(\xi))]^{v}$,

$Q_{\beta}(\xi)=Q(\xi)[B(\tilde{\eta}(\xi))]^{\beta}$

eşitlikleri sıklıkla kullanılacaktır. Burada $B(\tilde{\eta}(\xi))=\int_{\xi_{1}}^{\tilde{\eta}(\xi)} \tilde{a}^{-1 / \gamma}(s) d s$ şeklindedir.

$\mathrm{Bu}$ çalışmada incelenecek olan denklemin $\alpha=1$ durumu daha önce $\mathrm{Li}$ ve arkadaşları [15] tarafindan incelenmiştir. Çalışmalarında ikinci mertebeden nötral diferensiyel denklem için karşılaştırma teoremlerinden yararlanarak bazı salınımlılık kriterleri elde etmişlerdir. 


\subsection{Lemma [12]:}

$\left(H_{2}\right)$ ve $\left(H_{4}\right)$ sağlansın. Ayrıca

$\tilde{\tau}(\xi)=y\left(\tau\left(y^{-1}(\xi)\right)\right), \quad \tilde{\sigma}(\xi)=y\left(\sigma\left(y^{-1}(\xi)\right)\right)$

şeklinde tanımlı $\tilde{\tau}(\xi), \tilde{\sigma}(\xi)$ fonksiyonlar olsun. Bu durumda

$$
x(\tau(t))=\tilde{x}(\tilde{\tau}(\xi)), x(\sigma(t))=\tilde{x}(\tilde{\sigma}(\xi))
$$

sağlanır. Ve yeni bir şart olarak

$\left(H_{2}^{*}\right): \tilde{\tau}^{\prime}(\xi) \geq \tau_{0} l^{1-\alpha}=\tilde{\tau}_{0}>0, \tilde{\tau} \circ \tilde{\sigma}=\tilde{\sigma} \circ \tilde{\tau}$, $\tilde{\eta}(\xi) \leq \tilde{\sigma}(\xi)$ ve $\lim _{\xi \rightarrow+\infty} \tilde{\eta}(\xi)=+\infty$ olacak şekilde bir $\tilde{\eta}(\xi)$ fonksiyonu vardır,

yazılabilir.

\subsection{Lemma [13]:}

$x$, (1) denkleminin eninde sonunda pozitif bir çözümü ve $t \rightarrow \infty$ için $B(t) \rightarrow \infty$ olacak şekilde yeterince büyük bir $t_{1}$ varsa bu durumda,

$$
\begin{aligned}
& z(t)>0 ; \quad a(t)\left[D_{t}^{\alpha} z(t)\right]^{\gamma}>0 ; \\
& D_{t}^{\alpha}\left(a(t)\left[D_{t}^{\alpha} z(t)\right]^{\gamma}\right)<0,
\end{aligned}
$$

eşitsizlikleri eninde sonunda sağlanır.

\section{SALINIMLILIK}

$\mathrm{Bu}$ bölümde (1) denkleminin çözümlerinin salınımlılığ 1 incelenecektir.

\subsection{Teorem:}

$0<\beta \leq 1$ ve $\eta(t) \leq t \leq \tau(t)$ olsun. $\left(H_{1}\right)-($ $\left.H_{3}\right)$ ve $\left(H_{2}^{*}\right)$ koşulları sağlansın. Ayrıca $v \leq \beta$ ve $v<\gamma$ olacak şekilde iki tek doğal sayının oranı olan $v$ sayısının var olduğunu varsayalım. Bu durumda eğer

$$
\int_{\xi_{0}}^{\infty} Q_{v}(s) d s=\infty
$$

sağlanıyorsa (1) denklemi salınımlıdır.

\section{İspat:}

Aksine (1) denkleminin salınımlı olmadığını kabul edelim. Bu durumda denklem eninde sonunda işaret değiştirmeyen çözümlere sahiptir. Genelliği kaybetmeksizin, $\left[t_{1}, \infty\right)$ üzerinde (1) denkleminin bir $x(t)$ pozitif çözümünü göz önüne alalım (negatif çözümde benzerdir). Buna denk olarak $\left[\xi_{1}, \infty\right)$ üzerinde $\tilde{x}(\xi)$ alınabilir. Bu durumda [13, Theorem 3.1] in ispatındaki gibi,

$$
\left.\begin{array}{rl}
\left(\tilde{a}(\xi)\left[\tilde{z}^{\prime}(\xi)\right]^{\gamma}\right. & \left.\left.+\frac{p_{0}^{\beta}}{\tau_{0}} \tilde{a}(\tilde{\tau}(\xi))\left[\tilde{z}^{\prime}(\xi)\right]^{\gamma}\right)^{\prime}\right\} \\
& +Q(\xi) \tilde{z}^{\beta}(\tilde{\sigma}(\xi)) \leq 0
\end{array}\right\}
$$

eşitsizliği elde edilebilir. $\mathrm{Bu}$ eşitsizlik her $\xi \geq \xi_{1}$ ve bazı $\xi_{1} \geq \xi_{0}$ için sağlanır. Lemma 1 . $2 \operatorname{den} \tilde{z}^{\prime}(\xi)>0$ olduğunu biliyoruz. Böylece (3) ve $\tilde{\eta}(\xi) \leq \tilde{\sigma}(\xi)$ şartından

$$
\begin{aligned}
\left(\tilde{a}(\xi)\left[\tilde{z}^{\prime}(\xi)\right]^{\gamma}+\right. & \left.\frac{p_{0}^{\beta}}{\tau_{0}} \tilde{a}(\tilde{\tau}(\xi))\left[\tilde{z}^{\prime}(\xi)\right]^{\gamma}\right)^{\prime} \\
& +Q(\xi) \tilde{z}^{\beta}(\tilde{\eta}(\xi)) \leq 0
\end{aligned}
$$

yazılır. Ayrica $\tilde{z}(\xi)$ fonksiyonunun monotonluğundan ve $M>0$ sayısından

$$
\begin{aligned}
\tilde{z}^{\beta}(\tilde{\eta}(\xi)) & =\tilde{z}^{\beta-v}(\tilde{\eta}(\xi)) \tilde{z}^{v}(\tilde{\eta}(\xi)) \\
& \geq M^{\beta-v} \tilde{z}^{v}(\tilde{\eta}(\xi))
\end{aligned}
$$

olacağından

$$
\begin{gathered}
\left(\tilde{a}(\xi)\left[\tilde{z}^{\prime}(\xi)\right]^{\gamma}+\frac{p_{0}^{\beta}}{\tau_{0}} \tilde{a}(\tilde{\tau}(\xi))\left[\tilde{z}^{\prime}(\xi)\right]^{\gamma}\right)^{\prime} \\
+M^{\beta-v} Q(\xi) \tilde{z}^{v}(\tilde{\eta}(\xi)) \leq 0
\end{gathered}
$$

elde edilir. Burada,

$$
\tilde{w}(\xi)=\tilde{a}(\xi)\left[\tilde{z}^{\prime}(\xi)\right]^{\gamma}
$$

olarak tanımlanırsa, Lemma 1. 2 den, $\tilde{w}(\xi)$ nin pozitif ve azalan bir fonksiyon olduğunu söylenebilir. Bu yüzden 


$$
\tilde{z}(\xi) \geq \tilde{a}^{1 / \gamma}(\xi) \tilde{z}^{\prime}(\xi) B(\xi)=\tilde{w}^{1 / \gamma}(\xi) B(\xi)
$$

yazılır. $\mathrm{Bu}$ eşitsizlik (4) eşitsizliğinde kullanilırsa,

$$
\left.\begin{array}{l}
\left(\tilde{w}(\xi)+\frac{p_{0}^{\beta}}{\tau_{0}} \tilde{w}(\tilde{\tau}(\xi))\right)^{\prime} \\
+M^{\beta-v} Q_{v}(\xi) \tilde{w}^{v / \gamma}(\tilde{\eta}(\xi)) \leq 0
\end{array}\right\}
$$

$\tilde{w}(\xi)$ nin yukarıda elde edilen (5) gecikmeli diferensiyel eşitsizliğinin bir pozitif çözümü olduğunu görülür. Ayrica $\tilde{u}(\xi)$ fonksiyonu

$$
\tilde{u}(\xi)=\tilde{w}(\xi)+\frac{p_{0}^{\beta}}{\tau_{0}} \tilde{w}(\tilde{\tau}(\xi))
$$

ile tanımlanırsa ve $\tilde{w}(\xi)$ fonksiyonunun azalığından

$$
\tilde{u}(\xi) \leq \tilde{w}(\xi)\left(1+\frac{p_{0}^{\beta}}{\tau_{0}}\right)
$$

yazılır. $\mathrm{Bu}$ eşitsizlik (5) eşitsizliğinde kullanilırsa,

$$
\tilde{u}^{\prime}(\xi)+\left(\begin{array}{l}
M^{\beta-v}\left(\frac{\tilde{\tau}_{0}}{\tilde{\tau}_{0}+p_{0}^{\beta}}\right)^{v / \gamma} \\
\times Q_{v}(\xi) \tilde{u}^{v / \gamma}(\tilde{\eta}(\xi))
\end{array}\right) \leq 0
$$

Bulunur. Burada $\tilde{u}(\xi)$ nin, (6) gecikmeli diferensiyel eşitsizliğinin bir pozitif çözümü olduğu görülür.

Böylece [16, Theorem 1] den

$$
\tilde{u}^{\prime}(\xi)+\left(\begin{array}{c}
M^{\beta-v}\left(\frac{\tilde{\tau}_{0}}{\tilde{\tau}_{0}+p_{0}^{\beta}}\right)^{v / \gamma} \\
\times Q_{v}(\xi) \tilde{u}^{v / \gamma}(\tilde{\eta}(\xi))
\end{array}\right)=0
$$

bir pozitif çözüme sahiptir. Bu durumda [17, Theorem 2] den (2) nin kabulüyle (7) denkleminin salınımlı olduğunu ifade eder. Böylece (1) denklemi pozitif çözümlere sahip olamaz. $\mathrm{Bu}$ da kabulümüzle çelişir. İspat tamamlanır.

\subsection{Teorem:}

$0<\beta=\gamma \leq 1$ ve $\eta(t) \leq t \leq \tau(t)$ sağlansın.

Eğer $\left(H_{1}\right)-\left(H_{3}\right)$ şartları ve

$$
\frac{\tilde{\tau}_{0}}{\tilde{\tau}_{0}+p_{0}^{\beta}} \liminf _{\xi \rightarrow \infty} \int_{\tilde{\eta}(\xi)}^{\xi} Q_{\beta}(s) d s>\frac{1}{e}
$$

sağlanıyorsa, (1) denklemi salınımlıdır.

\section{İspat:}

Aksine (1) denkleminin salınımlı olmadığını kabul edelim. $\mathrm{Bu}$ durumda denklem eninde sonunda işaret değiştirmeyen çözümlere sahiptir. Genelliği kaybetmeksizin, $\left[t_{1}, \infty\right)$ üzerinde (1) denkleminin bir $x$ pozitif çözümünü göz önüne alalım. Bu durumda, Teorem 2.1 in ispatındakine benzer işlemlerle,

$$
\tilde{u}^{\prime}(\xi)+\frac{\tilde{\tau}_{0}}{\tilde{\tau}_{0}+p_{0}^{\beta}} Q_{\beta}(\xi) \tilde{u}(\tilde{\eta}(\xi))=0
$$

$\tilde{u}(\xi)$ pozitif çözüme sahip gecikmeli diferensiyel denklemi elde edilebilir. Diğer yandan (8) şartı ve [13, Lemma 3.4] den (9) denkleminin salınımlı olduğu sonucuna ulaşılabilir. Böylece çelişki elde edilir. Bu da ispat1 tamamlar.

\subsection{Sonuç:}

$0<\beta \leq 1, \beta \leq \gamma$ ve $\sigma(t)<t \leq \tau(t)$ olsun. Ayrıca $\left(H_{1}\right)-\left(H_{3}\right)$ şartları ve

$$
\left.\begin{array}{r}
\left(\frac{\tilde{\tau}_{0}}{\tilde{\tau}_{0}+p_{0}^{\beta}}\right)^{\beta / \gamma} \liminf _{\xi \rightarrow \infty} \int_{\tilde{\sigma}(\xi)}^{\xi} Q(s) \\
\times\left(\int_{\xi_{1}}^{\tilde{\sigma}(\xi)} \frac{d \zeta}{\tilde{a}^{1 / \gamma}(\zeta)}\right)^{\beta} d s>\frac{1}{e}
\end{array}\right\}
$$

eşitsizliği sağlanıyorsa, bu durumda denklemi salınımlıdır.

\subsection{Teorem:}

$0<\beta \leq 1$ ve $\eta(t)<\tau(t) \leq t$ olsun. $\left(H_{1}\right)-($

$\left.H_{3}\right)$ ve $\left(H_{2}^{*}\right)$ koşulları sağlansın. Ayrıca 
$v \leq \beta$ ve $v<\gamma$ olacak şekilde iki tek doğal sayının oranı olan $v$ sayısının var olduğunu varsayalım. Bu durumda eğer (2) sağlanıyorsa, (1) denklemi salınımlıdır.

\section{İspat:}

Aksine (1) denkleminin salınımlı olmadığını kabul edelim. Bu durumda denklem eninde sonunda işaret değiştirmeyen çözümlere sahiptir. Genelliği kaybetmeksizin, $\left[t_{1}, \infty\right)$ üzerinde (1) denkleminin bir $x$ pozitif çözümünü göz önüne alalım. Bu durumda $\begin{array}{llll}\text { Teorem } & 2.1 & \text { in } & \text { ispatinda }\end{array}$ $\tilde{w}(\xi)=\tilde{a}(\xi)\left[\tilde{z}^{\prime}(\xi)\right]^{\gamma}$ ile tanımlanan $\tilde{w}(\xi)$ fonksiyonu göz önüne alınabilir. Bu fonksiyon pozitiftir, azalandır ve (5) gecikmeli diferensiyel eşitsizliğini sağlamaktadır. Ayrıca $\tilde{u}(\xi)$ fonksiyonu tekrar göz önüne alınırsa, bu durumda $\tilde{w}(\xi)$ fonskiyonunun azalanlığından,

$$
\tilde{u}(\xi) \leq \tilde{w}(\tilde{\tau}(\xi))\left(1+\frac{p_{0}^{\beta}}{\tau_{0}}\right)
$$

elde edilir. $\mathrm{Bu}$ eşitsizlik (5) eşitsizliğinde yazılırsa, yeterince büyük $\xi$ için $\tilde{y}(\xi)$,

$$
\tilde{u}^{\prime}(\xi)+\left(\begin{array}{l}
M^{\beta-v}\left(\frac{\tilde{\tau}_{0}}{\tilde{\tau}_{0}+p_{0}^{\beta}}\right)^{v / \gamma} \\
\times Q_{v}(\xi) \tilde{u}^{v / \gamma}\left(\tilde{\tau}^{-1}(\tilde{\eta}(\xi))\right)
\end{array}\right) \leq 0
$$

gecikmeli diferensiyel eşitsizliğinin bir pozitif çözümüdür. Böylece [16, Theorem 1] den

$$
\tilde{u}^{\prime}(\xi)+\left(\begin{array}{l}
M^{\beta-v}\left(\frac{\tilde{\tau}_{0}}{\tilde{\tau}_{0}+p_{0}^{\beta}}\right)^{v / \gamma} \\
\times Q_{v}(\xi) \tilde{u}^{v / \gamma}\left(\tilde{\tau}^{-1}(\tilde{\eta}(\xi))\right)
\end{array}\right)=0
$$

bir pozitif çözüme sahiptir. Bu durumda (2) şartının [17, Theorem 2] de kullanılmasıyla (11) gecikmeli diferensiyel denklemi salınımlıdır. $\mathrm{Bu}$ da (11) denkleminin elde ettiğimiz $\tilde{y}(\xi)$ pozitif çözümüyle çelişir. $\mathrm{Bu}$ çelişki $x(t)$ nin (1) denkleminin salınımsız bir çözümü olmasından kaynaklandı, dolayısıyla ispat tamamlanır.

\subsection{Teorem:}

$0<\beta=\gamma \leq 1$ ve $\eta(t) \leq \tau(t) \leq t$ sağlansın.

Eğer $\left(H_{1}\right)-\left(H_{3}\right)$ şartları ve

$$
\frac{\tilde{\tau}_{0}}{\tilde{\tau}_{0}+p_{0}^{\beta}} \liminf _{\xi \rightarrow \infty} \int_{\tilde{\tau}^{-1}(\tilde{\eta}(\xi))}^{\xi} Q_{\beta}(s) d s>\frac{1}{e}
$$

eşitsizliği sağlanıyorsa (1) denklemi salınımlıdır.

\section{İspat:}

Teorem 2.4 ün ispatındaki benzer düşünce ve işlemlerle $\tilde{u}(\xi)$ nin,

$$
\tilde{u}^{\prime}(\xi)+\left(\begin{array}{c}
\frac{\tilde{\tau}_{0}}{\tilde{\tau}_{0}+p_{0}^{\beta}} Q_{\beta}(\xi) \\
\times \tilde{u}\left(\tilde{\tau}^{-1}(\tilde{\eta}(\xi))\right)
\end{array}\right)=0
$$

gecikmeli diferensiyel denkleminin eninde sonunda bir pozitif çözümü olduğu gösterilebilir. Ayrıca (8) şartı ve [13, Lemma 3.4] den (13) denkleminin salınımlı bir denklem olduğu görülür. $\mathrm{Bu}$ çelişki ispatı tamamlar.

\section{UYGULAMALAR}

$t \geq 1$ için aşağıdaki kesirli nötral diferensiyel denklem dikkate alınırsa,

$$
\left.\begin{array}{r}
\left.D_{t}^{1 / 3}\left(\left(D_{t}^{1 / 3}\left(x(t)+\frac{1}{t} x(\phi t)\right)\right)\right)\right) \\
+t x^{1 / 3}(\lambda t)=0
\end{array}\right\}
$$

(1) denklemine karşılık olarak, $a(t)=1$, $p(t)=\frac{1}{t}, \quad q(t)=t, \quad \phi \geq 1, \quad \tau(t)=\phi t$, $\lambda \in(0, \infty), \quad \sigma(t)=\lambda t, \quad \alpha=\frac{1}{3}, \quad \gamma=1$, $v=\beta=1, \quad \lambda_{1} \in(0,1), \quad \eta(t)=\lambda_{1} t \leq \lambda t$ yazılır. Ayrica 


$$
\begin{aligned}
& \xi=y(t)=\frac{t^{1 / 3}}{\Gamma(4 / 3)}, \quad y^{-1}(\xi)=\xi^{3} \Gamma^{3}(4 / 3), \\
& \xi_{1}=\frac{1}{\Gamma(4 / 3)}
\end{aligned}
$$

olacağından,

$$
\begin{aligned}
& \tilde{a}(\xi)=a\left(y^{-1}(\xi)\right)=1, \\
& \tilde{\sigma}(\xi)=y\left(\sigma\left(y^{-1}(\xi)\right)\right)=\frac{\left(\phi \xi^{3} \Gamma^{3}(4 / 3)\right)^{1 / 3}}{\Gamma(4 / 3)}=\phi^{1 / 3} \xi \\
& \tilde{q}(\xi)=q\left(y^{-1}(\xi)\right)=\xi^{3} \Gamma^{3}(4 / 3),
\end{aligned}
$$

yazılır. Dikkat edilirse $\left(H_{1}\right)-\left(H_{3}\right)$ şartları sağlanır ve

$$
\begin{aligned}
& 0 \leq p(t)=\frac{1}{t} \leq 1=p_{0}, \\
& \tau_{0}=(\phi t)^{\prime}=\phi, \\
& \int_{t_{0}}^{\infty} a^{-1 / \lambda}(s) d s=\infty, \\
& \lim _{t \rightarrow \infty} \frac{t}{\tau(t)}=\frac{1}{\phi}=l, \\
& \tilde{\tau}_{0}=\tau_{0} l^{1-1 / 3}=\phi^{1 / 3}
\end{aligned}
$$
$t \leq \tau(t)$ olduğundan

$$
\begin{aligned}
Q(t)= & \tilde{q}(\xi)=\xi^{3} \Gamma^{3}(4 / 3), \\
Q_{v}(\xi) & =Q(\xi)\left[\int_{\xi_{1}}^{\tilde{\eta}(\xi)} \tilde{a}^{-1 / \gamma}(s) d s\right]^{v} \\
& =\xi^{3} \Gamma^{3}(4 / 3)\left(\lambda_{1} \xi^{3} \Gamma^{3}(4 / 3)-\xi_{1}\right)^{1 / 3},
\end{aligned}
$$

elde edilir. Ayrıca,

$$
\int_{\xi_{1}}^{\infty} s^{3} \Gamma^{3}(4 / 3)\left(\lambda_{1} s^{3} \Gamma^{3}(4 / 3)-\xi_{1}\right)^{1 / 3} d s=\infty,
$$

olacağından Teorem 2.1 den dolayı (14) denklemi salınımlıdır.

\section{KAYNAKLAR}

[1] Kiryakova, V., Generalized fractional calculus and applications, Longman Group UK Limited, Essex (1994).

2] Magin, R. L. (2006). Fractional calculus in bioengineering (pp. 269-355). Redding: Begell House.

B Bai, Z., \& Xu, R. (2018). The Asymptotic Behavior of Solutions for a Class of Nonlinear Fractional Difference Equations with Damping Term. Discrete Dynamics in Nature and Society, 2018.

Bayram, M., Adiguzel, H., \& Ogrekci, S. (2015). Oscillation of fractional order functional differential equations with nonlinear damping. Open Physics, 13(1).

Agarwal, R. P., Lakshmikantham, V., \& Nieto, J. J. (2010). On the concept of solution for fractional differential equations with uncertainty. Nonlinear Analysis: Theory, Methods \& Applications, 72(6), 2859-2862.

Secer, A., \& Adiguzel, H. (2016). Oscillation of solutions for a class of nonlinear fractional difference equations. The Journal of Nonlinear Science and Applications (JNSA), 9(11), 58625869.

Chen, D. X. (2012). Oscillation criteria of fractional differential equations. Advances in Difference Equations, 2012(1), 33.

Matignon, D. (1996, July). Stability results for fractional differential equations with applications to control processing. In Computational engineering in systems applications (Vol. 2, pp. 963-968). Lille, France: IMACS, IEEE-SMC.

Öğrekçi, S. (2015). Generalized Taylor series method for solving nonlinear fractional differential equations with modified RiemannLiouville derivative. Advances in Mathematical Physics, 2015.

Öğrekçi, S. (2015). Interval oscillation criteria for functional differential equations of fractional order. Advances in Difference Equations, 2015(1), 3.

Muthulakshmi, V., \& Pavithra, S. (2017). Interval Oscillation Criteria for Forced Fractional Differential Equations with Mixed Nonlinearities. Global Journal of Pure and Applied Mathematics, 13(9), 6343-6353.

Wang, Y. Z., Han, Z. L., Zhao, P., \& Sun, S. R. (2015). Oscillation theorems for fractional neutral differential equations. Hacettepe journal of mathematics and statistics, 44(6), 14771488. 
[13] Ganesan, V., \& Kumar, M. S. (2016). Oscillation theorems for fractional order neutral differential equations. Journal of Applied Computer Science \& Mathematics (revised).

[14] Jumarie, G. (2006). Modified RiemannLiouville derivative and fractional Taylor series of nondifferentiable functions further results. Computers \& Mathematics with Applications, 51(9-10), 1367-1376.

[15] Li, T., \& Rogovchenko, Y. V. (2015). Oscillation of second-order neutral differential equations. Mathematische Nachrichten, 288(10), 1150-1162.

[16] Philos, C. G. (1981). On the existence of nonoscillatory solutions tending to zero at $\infty$ for differential equations with positive delays. Archiv der Mathematik, 36(1), 168-178.

[17] Kitamura, Y., \& Kusano, T. (1980). Oscillation of first-order nonlinear differential equations with deviating arguments. Proceedings of the American Mathematical Society, 64-68. 